\title{
Avaliação algimétrica por estímulo nociceptivo térmico e pressórico em cães pré-tratados com levomepromazina, midazolam e quetamina associados ou não ao butorfanol ou buprenorfina ${ }^{1}$
}

\author{
The algimetry evaluation for thermic and pressoric nociceptive stimulus in pre \\ treated dogs with methotrimeprazine, midazolam and ketamine associated or not with \\ butorphanol or buprenorphine
}

\section{André Leguthe Rosa ${ }^{2}$, Flávio Massone ${ }^{3}$}

1. Trabalho realizado no Departamento de Cirurgia e Anestesiologia Veterinária da Universidade Estadual Paulista (UNESP). Botucatu, São Paulo.

2. Prof. Dr. Universidade Metodista de São Paulo - Faculdade de Medicina Veterinária - São Bernardo do Campo/SP. Responsável pelo Serviço de Anestesiologia Veterinária - Centro Cirúrgico PROVET.

3. Prof. Titular da Faculdade de Medicina Veterinária da UNESP. Botucatu, São Paulo.

\section{RESUMO}

Objetivo: Quantificar a dor em cães sob anestesia dissociativa através de estímulo térmico e pressórico e o período hábil de dois analgésicos opióides. Métodos: Empregaram-se 30 cães alocados em três grupos $(\mathrm{n}=10)$, onde os animais de GI receberam levomepromazina e midazolam associado na mesma seringa à quetamina. Os animais de GII receberam o mesmo tratamento de GI porém associado ao butorfanol e por fim os animais de GIII receberam o mesmo tratamento de GI associando-se a buprenorfina. Procedeu se a avaliação paramétrica rotineira, empregando-se, entretanto, a termoalgimetria mensurada em ${ }^{\circ} \mathrm{C}$ em média de $52^{\circ} \mathrm{C}$ e a pressoalgimetria em kg. Resultados: Na termoalgimetria houve diferença significativa em GI nos momentos M0 e M1,e em M4 e M5. Em GII houve diferença em M0, M1, M5 e M6. Em GIII houve diferença entre momentos M0 e M1. Na pressoalgimetria houveram diferenças em GI em diferentes momentos: M0, M2 e M3. Em GII observaram-se diferenças em todos os momentos. Em GIII observaram-se diferenças em M0 e M9. Ocorreram diferenças entre os grupos, sendo o M2 de GII menor que GI e GIII. Já em M4 e M5 de GIII demonstrou-se maior que GI e GII. E na avaliação dos períodos observou-se um período de latência significativamente maior em GI, porém com período hábil e de recuperação menor em relação à GII e GIII. Já a recuperação do tônus postural foi maior em GIII seguido de GII e finalmente de GI. Conclusão: O método empregado para mensuração do estímulo álgico foi eficiente, observando-se um período hábil analgésico de 3 horas para o butorfanol e de 6 horas para a buprenorfina.

Descritores: Analgesia. Algimetria. Anestesia dissociativa. Butorfanol. Buprenorfina. Cão.

\section{ABSTRACT}

Purpose: This study aimed at quantifies the pain in dogs under dissociative anesthesia, across thermal and pressoric stimulus and quantify the reasonable period between two different opioids analgesics. Methods: In this study, 30 dogs were used and, divided into three groups of 10 animals each, in which the animals of GI received methotrimeprazine and midazolam put on the same syringe with ketamine. The animals of GII received the same treatment of GI but associated with butorphanol and finally the animals of GIII received the same treatment of GI but associated with buprenorphine. The routine parametric evaluations has been proceeded, although using the thermo algimetry measured in ${ }^{\circ} \mathrm{C}$ with the average of $52^{\circ} \mathrm{C}$ and the pressoric algimetry in Kg. Results: In the thermo algimetry, there has been significant difference in GI at the moments M0, M1, M4 
and M5; in GII it was found at M0, M1, M5 and M6 and in GIII it was observed the significant at M0 and M1. It has also been shown in pressoric algimetry significant difference in GI at the moments M0, M2 and M3. Among GII it has observed significant difference at all moments and it has found at M0, M9 in GIII. Thus, it has observed significant differences between all groups; for such the M2 of GII smaller than the others; and M4, M5 of GIII bigger than GI and GII. In the assessment of all periods it was observed significant latent period bigger in GI, however, with reasonable period and short recovery in GII and GIII. In the order hand, the postural tonus recovery it was longer in GIII, followed by GII and finally GI. Conclusion: The used method for the measurement of algic stimulus was efficient, noticing a reasonable analgesic period of 3 hours for butorphanol and 6 hours for buprenorphine.

Key words: Analgesia. Algimetry. Dissociative Anesthesia. Butorphanol. Buprenorfine. Dog.

\section{Introdução}

AAnestesiologia Veterinária desde fim do século passado se desenvolveu de forma célere, tanto na utilização de anestesia geral quanto a anestesia dissociativa em suas diversas associações ${ }^{1}$. A utilização de grupos farmacológicos específicos auxiliam nas associações, permitindo empregar os opióides de maneira mais segura, tornando as anestesias dissociativas mais técnicas e éticas. $\mathrm{O}$ butorfanol e a buprenorfina são analgésicos opióides que podem ser associados à anestesia dissociativa e são compatíveis na associação com levomepromazina e midazolam², o que tornaria a anestesia dissociativa uma opção à anestesia geral ${ }^{3}$.

A levomepromazina é uma fenotiazina pertencente à série mista, com ações adrenolítica e antihistamínicas $^{1}$, acrescida ainda de efeito analgésico ${ }^{4}$. Seus efeitos são semelhantes aos dos outros fármacos fenotiazínicos, que causam depressão acentuada do sistema nervoso central (SNC), agindo seletivamente em algumas regiões e atuando também na periferia ${ }^{4}$. A hipotensão arterial é seu principal efeito colateral ${ }^{5}$ e possui também características antiarrítmicas ${ }^{6,5}$. As doses variam de 0,5 a $2,0 \mathrm{mg} / \mathrm{kg}$ pelas vias intravenosa, intramuscular ou subcutânea porém, a dose de $1,0 \mathrm{mg} / \mathrm{kg}$ é suficiente para causar tranquilização e potencializar o efeito dos barbitúricos em $50 \%{ }^{1}$.

O midazolam, rotineiramente é usado na associação com fenotiazina na dose de $0,2 \mathrm{mg} / \mathrm{kg}^{1} \mathrm{e}$ não altera significativamente a freqüência cardíaca e temperatura retal elevando discretamente a freqüência respiratória.

A quetamina éum anestésico dissociativo de ação rápida que dissocia o córtex cerebral de maneira seletiva, estimulando a freqüência cardíaca, exercendo vasoconstrição periférica e resultando em aumento da pressão arterial, não alterando significativamente a freqüência respiratória, mantendo livre as vias aéreas e reflexos protetores, com redução do volume urinário 7.

Tradicionalmente os opióides são utilizados para proporcionar analgesia, porém apresentam o inconveniente de promover depressão respiratória dose-dependente e aumentar a sedação, prolongando assim o tempo de recuperação ${ }^{8}$. O butorfanol foi descrito como um derivado sintético da morfina sendo de quatro a sete vezes mais potente ${ }^{9}$. A depressão respiratória máxima ocorre com doses acima de 4 mg em pacientes caninos adultos. Um efeito-teto similar também ocorre para a analgesia. O butorfanol é eficaz como suplemento durante anestesia balanceada e causa menor depressão respiratória pós-operatória que a morfina ${ }^{10}$.

Da mesma maneira que o butorfanol pode ser asssociado em anestesia injetável balanceada ${ }^{10}$, a buprenorfina é também uma alternativa para melhorar a qualidade desta modalidade anestésica ${ }^{11}$.

A buprenorfina foi descrita como sendo um derivado semi-sintético da tebaína; é um analgésico potente e pode ser administrado pela via intravenosa ou intramuscular, obtendo-se um efeito total lento após administração intravenosa, entretanto uma vez estabelecida a analgesia é mantida por um período prolongado ${ }^{11}$. A buprenorfina, pode ser utilizada pelas vias intravenosa, intramuscular ou subcutânea dez à quinze minutos antes do término da cirurgia. O efeito analgésico da buprenorfina é cerca de trinta vezes mais potente que a morfina e sua duração é em torno de oito horas ${ }^{4}$. 


\section{Fisiopatologia da dor}

A nocicepção é a tradução, condução e o processamento central dos sinais recebidos geralmente por estimulação dos nociceptores. Este é um processo que quando ocorre resulta na percepção consciente da dor ${ }^{12}$. Os estímulos gerados são captados pelos nociceptores, conduzidos por fibras aferentes, interneurônios e medula espinhal, chegando ao hipotálamo, córtex cerebral e sistema límbico, onde a dor é reconhecida, em termos de localização, natureza e intensidade ${ }^{13,14}$.

Atualmente, não há métodos objetivos para avaliação da intensidade da dor, tanto em pacientes humanos quanto veterinários; sendo em animais ainda mais subjetiva ${ }^{13}$. Desta forma, a avaliação da mesma requer uma observação cuidadosa do paciente ${ }^{15,16}$. Entretanto, somando-se as respostas comportamentais, hormonais e metabólicas ${ }^{17}$, podese obter uma avaliação com maior tendência à realidade e intensidade da dor.

\section{Métodos}

Foram empregados 30 cães machos ou fêmeas com peso variando de 8,5 a 17,0 quilos provindos do Biotério Central da UNESP - campus de Botucatu. Tomamos a precaução de não utilizar no experimento animais em prenhes, cio, idosos e não clinicamente sadios, e distribuídos aleatoriamente em três grupos. No grupo I (G I) os animais foram submetidos a jejum alimentar prévio de 6 horas e hídrico de 3 horas. Foram conduzidos na sala experimental uma hora antes do início da pesquisa a fim de se adaptarem ao meio. Após a avaliação paramétrica rotineira os animais receberam a medicação pré-anestésica (MPA) com levomepromazina pela via intravenosa e decorridos 15 minutos receberam pela via intramuscular a associação de midazolam e quetamina ambas na mesma seringa.

No grupo II (GII) seguiu-se o mesmo protocolo experimental de GI só que na aplicação intramuscular associou-se midazolam, quetamina ao butorfanol (os três na mesma seringa).

No grupo III (GIII) seguiu-se o mesmo protocolo experimental de GI só que na aplicação intramuscular associou-se midazolam, quetamina à buprenorfina (os três na mesma seringa).
As doses e vias correspondentes de administração foram: Levomepromazina a 1,0 mg/ $\mathrm{kg}$ (via intravenosa), Midazolam a 0,2 mg/kg (via intramuscular), Quetamina a 15,0 mg/kg (via intramuscular), Butorfanol a 0,2 mg/kg (via intramuscular) e Buprenorfina a $6,0 \mathrm{mg} / \mathrm{kg}$ (via intramuscular).

Os momentos empregados para a avaliação paramétrica foram: M0 (início do experimento e imediatamente antes de receber a levomepromazina), M1 (após 15 minutos da aplicação da medicação pré-anestésica e aplicação imediata da associação anestésica estabelecida para o grupo (via intramuscular), M2 (após 30 minutos de M0), M3 (após 45 minutos de M0), M4 (após 60 minutos de M0), M5 (após 120 minutos de M0), M6 (após 180 minutos de M0), M7 (após 240 minutos de M0), M8 (após 300 minutos de M0) e M9(após 360 minutos de M0).

Os atributos avaliados foram: algimetria em escala crescente de 52 a $60^{\circ} \mathrm{C}^{6}$ Termo Algimetro IOPE,Instr. de precisão ltda; algimetria quantificada de maneira pressórica em Kg; os períodos de latência, hábil anestésico e de recuperação; e tônus postural.

A mensuração álgica foi avaliada por aparelhos com tecnologia idealizada pelos docentes da Anestesiologia Experimental empregando a termometria e mensuração pressórica de arraste através de tecnologia desenvolvida por bioengenharia nacional, esses dados foram obtidos aplicando-se os aparelhos para estímulo na prega interdigital dos modelos experimentais (Figuras 1 e 2). Os demais dados foram analisados pelo aparelho multiparamétrico já existentena sala de Anestesiologia Experimental onde se realizou a pesquisa em questão. Cada variável foi estudada individualmente, e para as variáveis de período de latência, período hábil, período de recuperação e tônus postural, que apresentaram distribuição normal, foi utilizada a Análise de Variância seguida do método de Tukey. Para as variáveis termoalgimetria e pressoalgimetria, para comparação dos momentos dentro de cada grupo, foi utilizado o teste não paramétrico de Friedmann, seguido do teste de comparações múltiplas; e para comparação dos grupos dentro de cada momento foi utilizado o teste de Kruskal-Wallis, seguido do teste de comparações múltiplas, com nível de significância de 5\%. 


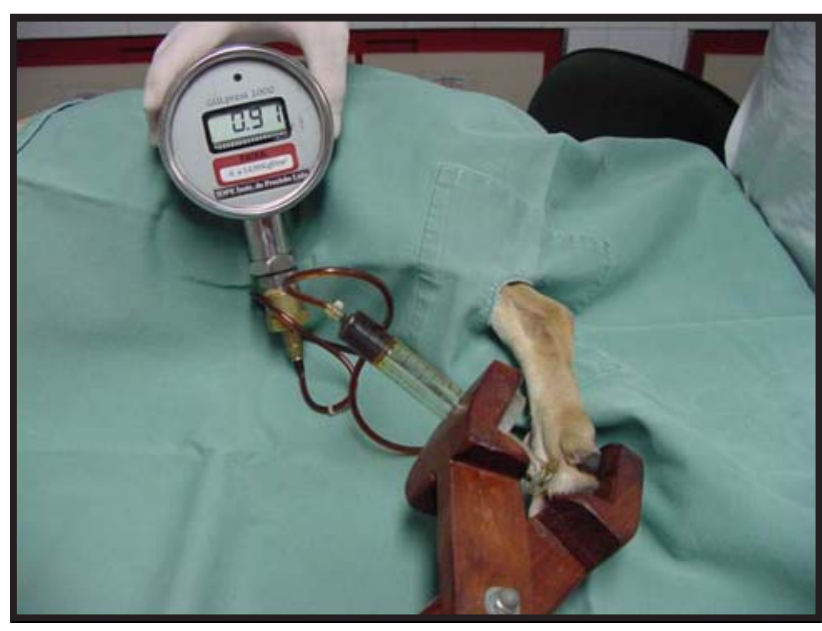

FIGURA 1 - Aplicação de pressoalgímetro na prega interdigital do modelo experimental

\section{Resultados}

A termoalgimetria evidenciou diferença entre os momentos dentro dos mesmos grupos, e foi demonstrada também diferença entre os grupos. Houve diferença significativa em GI nos momentos

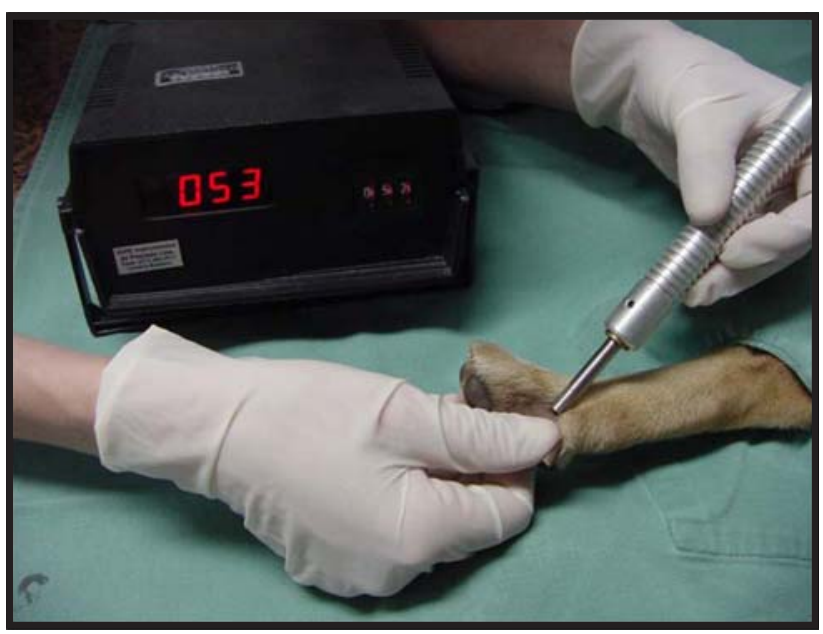

FIGURA 2 - Aplicação de termoalgímetro na prega interdigital do modelo experimental

M0 e M1(valores menores) e em M4 e M5. Em GII houve diferença significativa em M0, M1, M5 e M6 (valores menores). Em GIII houve diferenças entre momentos M0 e M1(valores menores) - (Tabela $1 \mathrm{e}$ Figura 3).

TABELA 1 - Valores medianos, $1^{\text {os }}$ e $3^{\text {os }}$ quartis da termoalgimetria em ${ }^{\circ} \mathrm{C}$, de cães $(\mathrm{n}=10)$, tratados com $1 \mathrm{mg} / \mathrm{kg}$ de levomepromazina, $0,2 \mathrm{mg} / \mathrm{kg}$ de midazolam e $15 \mathrm{mg} / \mathrm{kg}$ de quetamina (GI), prétratados com butorfanol (G II) ou pela buprenorfina (G III)

\begin{tabular}{lllllllllll}
\hline Momentos & M0 & M1 & M2 & M3 & M4 & M5 & M6 & M7 & M8 & M9 \\
\hline G I & $\mathbf{5 2 , 0}$ & $\mathbf{5 3 , 0}$ & $\mathbf{6 0 , 0}$ & $\mathbf{6 0 , 0}$ & $\mathbf{5 7 , 0}$ & $\mathbf{5 4 , 0}$ & & & & \\
Quartis & 52,0 & 53,0 & 60,0 & 60,0 & 56,0 & 52,0 & & & \\
Significância & 52,0 & 54,0 & 60,0 & 60,0 & 58,0 & 55,0 & & & & \\
& $\mathrm{a}^{(2)}$ & $\mathrm{aD}$ & $\mathrm{aA}$ & $\mathrm{aA}$ & $\mathrm{cB}$ & $\mathrm{bC}$ & & & & \\
& $\mathrm{E}^{(1)}$ & & & & & & & & & \\
G II & $\mathbf{5 2 , 0}$ & $\mathbf{5 2 , 0}$ & $\mathbf{6 0 , 0}$ & $\mathbf{6 0 , 0}$ & $\mathbf{6 0 , 0}$ & $\mathbf{5 6 , 0}$ & $\mathbf{5 2 , 0}$ & & & \\
Quartis & 52,0 & 52,0 & 60,0 & 60,0 & 55,0 & 52,0 & 52,0 & & & \\
Significância & $\mathrm{aC}$ & $\mathrm{aC}$ & $\mathrm{aA}$ & $\mathrm{aA}$ & $\mathrm{bA}$ & $\mathrm{bB}$ & $\mathrm{bC}$ & & & \\
G III & $\mathbf{5 2 , 0}$ & $\mathbf{5 4 , 0}$ & $\mathbf{6 0 , 0}$ & $\mathbf{6 0 , 0}$ & $\mathbf{6 0 , 0}$ & $\mathbf{6 0 , 0}$ & $\mathbf{6 0 , 0}$ & $\mathbf{6 0 , 0}$ & $\mathbf{6 0 , 0}$ & $\mathbf{5 5 , 0}$ \\
Quartis & 52,0 & 53,0 & 60,0 & 60,0 & 60,0 & 60,0 & 60,0 & 60,0 & 55,0 & 53,0 \\
& 52,0 & 54,0 & 60,0 & 60,0 & 60,0 & 60,0 & 60,0 & 60,0 & 60,0 & 59,0 \\
Significância & $\mathrm{aB}$ & $\mathrm{aB}$ & $\mathrm{aAB}$ & $\mathrm{aAB}$ & $\mathrm{aAB}$ & $\mathrm{aAB}$ & $\mathrm{aAB}$ & $\mathrm{A}$ & $\mathrm{A}$ & $\mathrm{AB}$ \\
\hline
\end{tabular}

Nota: não se dispõe de dados pois, para os Grupos I e II não foram feitas medidas nestes momentos.

(1) - Momentos representados pelas medianas dentro de cada grupo seguidas de mesma letra maiúscula não diferem estatisticamente $(\mathrm{p}>0,05)$

(2) - Momentos representados pelas medianas dentro de cada grupo seguidas de mesma letra minúscula não diferem estatisticamente $(\mathrm{p}>0,05)$

Válido para todas as tabelas. 
Termo-algimetria

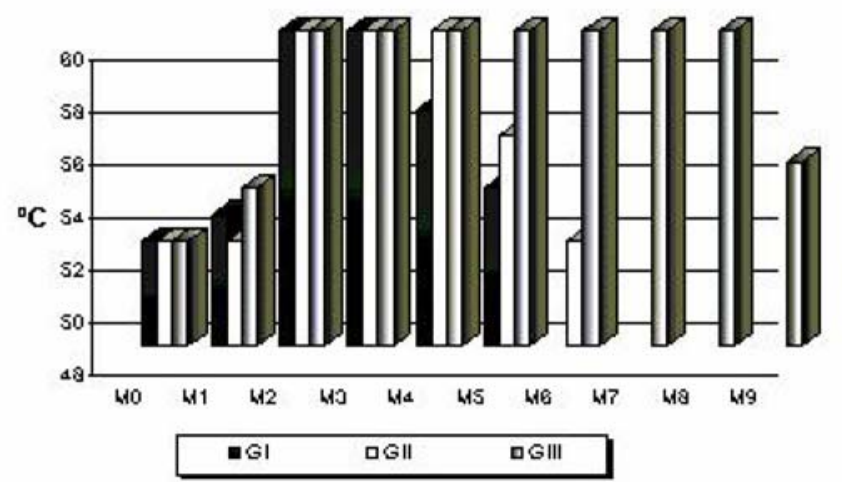

FIGURA 3 - Valores medianos da termoalgimetria em ${ }^{\circ} \mathrm{C}$, de cães $(\mathrm{n}=10)$, tratados com $1 \mathrm{mg} / \mathrm{kg}$ de levomepromazina, $0,2 \mathrm{mg} / \mathrm{kg}$ de midazolam e $15 \mathrm{mg} / \mathrm{kg}$ de quetamina (GI), pré-tratados com butorfanol (G II) ou pela buprenorfina (G III)

Na avaliação da pressoalgimetria observaramse diferenças em GI em diferentes momentos em M0 (menor valor) e M2 e M3 (maiores valores). Em GII observaram-se diferenças em M0, M1e M6 (valores menores) e M2, M3, M4 e M5 (valores maiores). Em GIII observaram-se diferenças em M0 e M9 (valores menores). Ocorreram diferenças entre os grupos, sendo o M2 de GII menor que GI e GIII.
Já em M4 e M5 de GIII demonstrou-se maior que GI e GII - (Tabela 2 e Figura 4).

$\mathrm{Na}$ avaliação dos períodos observou-se um período de latência significativamente maior em GI, porém com período hábil e de recuperação menor em relação à GII e GIII. Já a recuperação do tônus postural foi maior em GIII seguido de GII e finalmente de GI - (Tabela 3e Figura 5) .

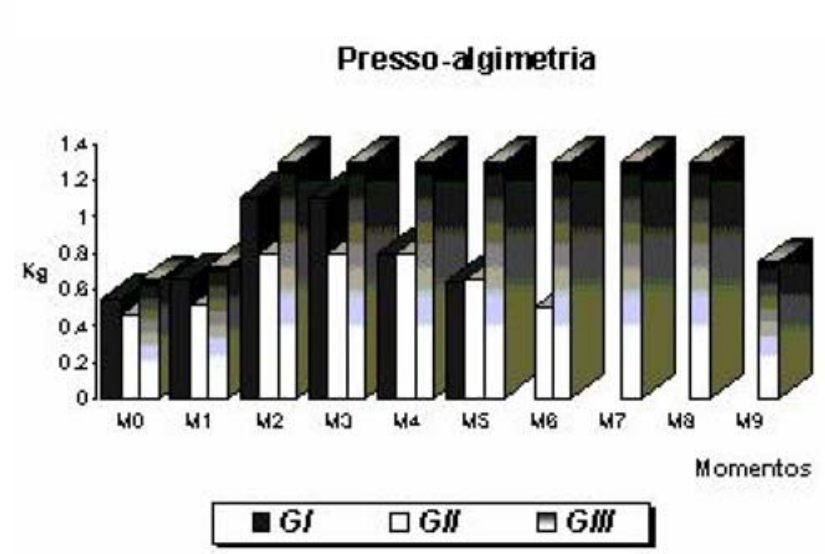

FIGURA 4 - Valores medianos da pressoalgimetria em $\mathrm{kg}$, de cães $(\mathrm{n}=10)$, tratados com $1 \mathrm{mg} / \mathrm{kg}$ de levomepromazina, $0,2 \mathrm{mg} / \mathrm{kg}$ de midazolam e $15 \mathrm{mg} / \mathrm{kg}$ de quetamina (GI), pré-tratados com butorfanol (G II) ou pela buprenorfina (G III)

TABELA 2 - Valores medianos, $1^{\text {os }}$ e $3^{\text {os }}$ quartis da pressoalgimetria em kg, de cães (n=10), tratados com 1 mg $/ \mathrm{kg}$ de levomepromazina, $0,2 \mathrm{mg} / \mathrm{kg}$ de midazolam e $15 \mathrm{mg} / \mathrm{kg}$ de quetamina (GI), pré-tratados com butorfanol (G II) ou pela buprenorfina (G III)

\begin{tabular}{|c|c|c|c|c|c|c|c|c|c|c|}
\hline Momentos & MO & M1 & M2 & M3 & M4 & M5 & M6 & M7 & M8 & M9 \\
\hline G I & 0,54 & 0,66 & 1,10 & 1,10 & 0,80 & 0,64 & & & & \\
\hline \multirow[t]{2}{*}{ Quartis } & 0,40 & 0,55 & 0,80 & 0,80 & 0,72 & 0,53 & & & & \\
\hline & 0,60 & 0,82 & 1,20 & 1,20 & 0,91 & 0,72 & & & & \\
\hline Significância & $a^{(2)} E^{(1)}$ & $A C$ & $\mathrm{aA}$ & $\mathrm{aA}$ & bB & bD & & & & \\
\hline G II & 0,46 & 0,51 & 0,80 & 0,80 & 0,80 & 0,65 & 0,50 & & & \\
\hline \multirow[t]{2}{*}{ Quartis } & 0,34 & 0,40 & 0,70 & 0,70 & 0,70 & 0,51 & 0,42 & & & \\
\hline & 0,55 & 0,62 & 1,10 & 1,10 & 1,10 & 0,81 & 0,58 & & & \\
\hline Significância & $\mathrm{aB}$ & $A B$ & bA & $\mathrm{aA}$ & bA & bA & bB & & & \\
\hline G III & 0,65 & 0,73 & 1,30 & 1,30 & 1,30 & 1,30 & 1,30 & 1,30 & 1,30 & 0,75 \\
\hline \multirow[t]{2}{*}{ Quartis } & 0,44 & 0,58 & 0,90 & 0,90 & 0,90 & 0,90 & 0,90 & 0,90 & 0,90 & 0,54 \\
\hline & 0,69 & 0,79 & 1,40 & 1,40 & 1,40 & 1,40 & 1,40 & 1,40 & 1,40 & 0,87 \\
\hline Significância & $\mathrm{aB}$ & $A A B$ & $\mathrm{aAB}$ & $\mathrm{aAB}$ & $\mathrm{aAB}$ & $\mathrm{aAB}$ & $\mathrm{aAB}$ & $A B$ & $A B$ & $A B$ \\
\hline
\end{tabular}

Nota: não se dispõe de dados pois, para os Grupos I e II não foram feitas medidas nestes momentos.

(1) - Momentos representados pelas medianas dentro de cada grupo seguidas de mesma letra maiúscula não diferem estatisticamente $(\mathrm{p}>0,05)$

(2) - Momentos representados pelas medianas dentro de cada grupo seguidas de mesma letra minúscula não diferem estatisticamente $(\mathrm{p}>0,05)$

Válido para todas as tabelas. 
TABELA 3 - Valores médios e desvios-padrão referentes ao período de latência, (PL) hábil anestésico (PHA) recuperação (PR) e tônus postural (TP) em minutos, de cães ( $\mathrm{n}=10)$ tratados com $1 \mathrm{mg} / \mathrm{kg}$ de levomepromazina, 0,2 mg/ kg de midazolam e $15 \mathrm{mg} / \mathrm{kg}$ de quetamina (GI), pré-tratados com butorfanol (G II) ou pela buprenorfina (G III)

\begin{tabular}{lllll}
\hline Períodos & Latência & Hábil & Recuperação & Tônus postural \\
\hline G I & $\mathbf{5 , 2 0}$ & $\mathbf{4 7 , 2 0}$ & $\mathbf{6 4 , 5 0}$ & $\mathbf{8 0 , 0}$ \\
S & 1,62 & 10,60 & 17,68 & $\mathbf{1 8 , 3 1}$ \\
Significância & $\mathrm{a}^{(1)}$ & $\mathrm{b}$ & $\mathrm{b}$ & $\mathrm{c}$ \\
G II & $\mathbf{3 , 5 0}$ & $\mathbf{8 0 , 4 0}$ & $\mathbf{1 0 6 , 2 0}$ & $\mathbf{1 2 8 , 9 0}$ \\
S & 1,08 & 19,82 & 21,21 & 22,64 \\
Significância & $\mathrm{b}$ & $\mathrm{a}$ & $\mathrm{a}$ & $\mathrm{b}$ \\
G III & $\mathbf{3 , 5 0}$ & $\mathbf{9 1 , 9 0}$ & $\mathbf{1 1 7 , 5 0}$ & $\mathbf{1 6 6 , 8 0}$ \\
S & 1,26 & 22,80 & 117,50 & 22,90 \\
Significância & $\mathrm{b}$ & $\mathrm{a}$ & $\mathrm{a}$ & $\mathrm{a}$ \\
\hline
\end{tabular}

(1) - Para cada variável, médias seguidas de mesma letra não diferem estatisticamente ( $\mathrm{p}>0,05)$.

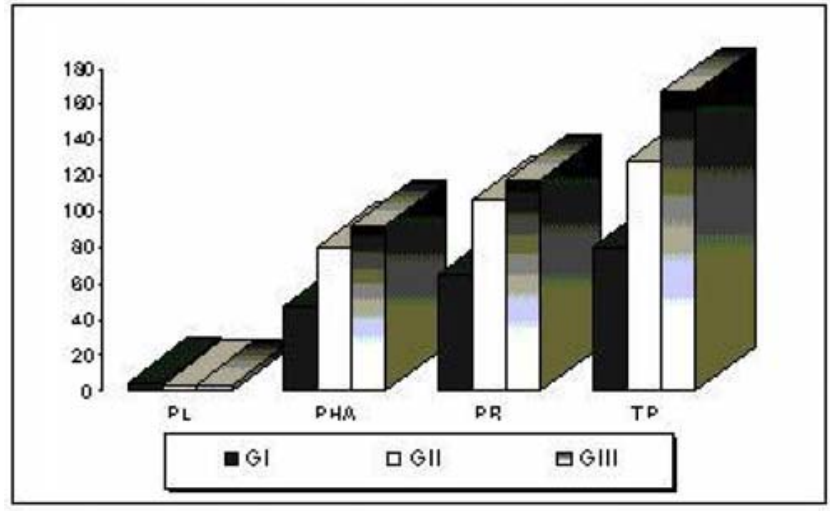

FIGURA 5 - Valores médios referentes às variáveis: período de latência, (PL) hábil anestésico ,(PHA) recuperação (PR) e tônus postural (TP) em minutos, de cães $(n=10)$ tratados com $1 \mathrm{mg} /$ $\mathrm{kg}$ de levomepromazina, 0,2 $\mathrm{mg} / \mathrm{kg}$ de midazolam e $15 \mathrm{mg} / \mathrm{kg}$ de quetamina (GI), prétratados com butorfanol (G II) ou pela buprenorfina (G III) segundo grupos

\section{Discussão}

Segundo Holton e $\operatorname{col}^{19}$., a dor é uma experiência pessoal que éúnica para cada indivíduo, e, atualmente, não existem métodos objetivos para avaliar a intensidade de dores clínicas, o que acaba resultando na utilização de métodos subjetivos para essas avaliações. E também associações com outras formas de avaliação, como as alterações fisiológicas e /ou metabólicas são necessárias para uma maior confiabilidade no resultados obtidos ${ }^{18}$.

São amplamente citados na literatura métodos de avaliação de dor como a escala descritiva simples
(SDS), escala de classificação numérica (NRS) e ainda a escala visual analógica (VAS), sendo esse último um método bastante empregado ${ }^{19,20}$.

Os métodos mais simples de usar o comportamento para avaliar a dor envolvem observação dos reflexos em resposta a um estímulo doloroso agudo, que pode ser elétrico, térmico ou mecânico, aplicado geralmente a cauda ou a pata e que resulta em movimento ou esquiva do local da aplicação do estímulo ${ }^{21}$. No presente estudo, os resultados obtidos, determinaram que os métodos utilizados foram eficazes para a avaliação do potencial analgésico dos fármacos empregados; sendo considerada analgesia a diminuição da sensação ou aumento na tolerância à dor ${ }^{22}$, e que foi observado no presente estudo. Observando então, as formas de avaliação da dor em cães ${ }^{18}$, acredita-se, que a mensuração e/ou quantificação da dor através da presso e da termo algimetria também foram eficientes na avaliação do período hábil de analgesia dos fármacos estudados, fato este também observado por outros autores ${ }^{23,24}$, onde relataram como período hábil analgésico para a buprenorfina e para o butorfanol dados bem próximos aqueles que foram observados neste estudo.

\section{Conclusão}

O sistema de mensuração e/ou avaliação da dor pelos sistemas de termoalgimetria e de pressoalgimetria, mostrou-se eficiente para quantificar o estímulo álgico; período de duração da analgesia dos analgésicos opióides, que foram de três horas para o butorfanol e de seis horas para a buprenorfina. 


\section{Referências}

1. Massone F. Anestesiologia veterinária: farmacologia e técnicas. São Paulo: Guanabara Koogan; 2003.

2. Ko JCH, Smith TA, Kuo WC. Comparison of anesthetic and cardiorespiratory effects of diazepam-butorphanolketamine, acepromazine-butorphanol-ketamine, and xylazine-butorphanol-ketamine in ferrets. J Am Animal Hosp Assoc. 1998;34:407-16.

3. Jacobson JD, McGrath CH, Smith EP. Cardiovascular effects of induction and maintenance of anesthesia with ketamine-midazolam combination, with and without prior administration of butorphanol or oxymorphone. Am J Vet Res. 1994;55:543-50.

4. Spinosa HS, Górniak SL, Bernardi MM. Farmacologia aplicada à medicina veterinária. Rio de Janeiro: Guanabara Koogan; 1999.

5. Muir III WW, Hubbell JAE, Skarda RT, Bednarski RM. Anestesia veterinária. São Paulo; 2001.

6. Short CE. Principles and practice of veterinary anesthesia. Baltimore: Willians \& Wilkins CO; 1987.

7. Vianna PTG. Efeitos da quetamina sobre a função renal e eletrólitos (sódio e potássio) [Tese Doutorado].Universidade Estadual Paulista - Faculdade de Medicina; 1980.

8. Fantoni D. Estudo comparativo entre o ketoprofeno e o flinixin meglumine no tratamento da dor pós-operatória de cães submetidos à cirurgia ortopédica. Clin Vet. 1999;19:19-22.

9. Trim CM. Cardiopulmonary effects of butorphanol tartrate in dogs. Am J Vet Res. 1983;44:329-31.

10. Troncy E, Besner JG, Charbonneau R, Cuvelliez SG, Blais D. Pharmacokinetics of epidural butorphanol in isoflurane-anesthetized dogs. J Vet Pharmacol Therap. 1996;19:268-73.

11. Brodbelt DC, Taylor PM, Stanway GW. A comparison of preoperative morphine and buprenorphine for postoperative analgesia for arthotomy in dogs. J Vet Pharmacol Therap.1997;20:284-9.

12. Thurmon JC, Tranquilli WJ, Benson GJ. Essentials of small animal anesthesia and analgesia. Philadelphia: Copyright; 1999.

13. Sackman JE. Pain: its perception and alleviation in dogs and cats. Pat I. The physiology of pain. Compend Cont Educ Pract Vet. 1991;1:35-40.

14. Livingston A. Physiological basis for pain perception in animals. J Vet Anaesth. 1994;21:73-81.

15. Taylor PM, Houlton JEF. Post-operative analgesia in the dog: a comparison of morphine, buprenorphine and pentazocine. J Small Anim Pract. 1984;25:437-51.

16. Reid J, Nolan AM, Welsh EM. Estudos recentes sobre o uso de flunixin meglumine em cães. Hora Vet. 1995;86:1721.

17. Janig W. The sympathetic nervous system in pain. Eur Compend J Anaesth. 1995;12:53-60.

18. Mathews KA, Paley DM, Foster RA. A comparison of ketorolac with flunixin, butorphanol, and oxymorphone in controlling postoperative pain in dogs. Can Vet J. 1996;37:557-67.

19. Holton LL, Scott EM, Nolan AM. Comparison of three methods used for assessment of pain in dogs. J Am Vet Med Assoc. 1998;212:61-6.

20. Grimm KA, Tranquilli WJ, Thurmon JC. Duration of nonresponse to noxius stimulation after intramuscular administration of butorphanol, medetomidine, or a butorphanol-medetomidine combination during isoflurane administration in dogs. Am J Vet Res. 2000;61:42-7.

21. Dubner R. Methods of assessing pain in animals. In: Wall PD, Melzack R. Textbook of pain. 3ed. Edinburgh: Churchill Livingstone; 1994. p.293-302.

22. Sawyer DC, Rech RH, Adams T. Analgesia and behavioral responses of dogs given oxymorphoneacepromazine and meperidine-acepromazine after methoxyflurane and halothane anesthesia. Am J Vet Res. 1992;53:1361-8.

23. Souza HJM. Avaliação e controle da dor em felinos. Anais II Congresso Internacional da Medicina Felina. Rio de Janeiro; 2001.

24. Morgan GE, Mikhail MS. Clinical anesthesiology. Stamford: Copyright; 1996.
Correspondência:

André Leguthe Rosa

Centro Cirúrgico PROVET

Av. Jurucê, 903

04080-013 São Paulo - SP

Tel: (11) 5052-5909

Fax:(11) 5055-9271

leguthe@terra.com.br
Conflito de interesse: nenhum

Fonte de financiamento: nenhuma

Recebimento: 25/09/2004

Revisão: 04/10/2004

Aprovação: 20/11/2004

Como citar este artigo:

Rosa AL, Massone F. Avaliação algimétrica por estímulo nociceptivo térmico e pressórico em cães pré-tratados com levomepromazina, midazolam e quetamina associados ou não ao butorfanol ou buprenorfina. Acta Cir Bras. [serial online] 2005 Jan-Fev;20(1). Disponível em URL: http://www.scielo.br/acb

*Figuras coloridas disponíveis em http://www.scielo.br/acb 